\title{
Correctly rounded multiplication by arbitrary precision constants
}

\author{
Nicolas Brisebarre \\ Laboratoire LARAL, Université Jean Monnet (Saint-Étienne) \\ and Laboratoire LIP \\ Jean-Michel Muller \\ CNRS, Laboratoire LIP (CNRS/ENS Lyon/INRIA/Univ. Lyon 1), \\ Projet Arénaire, 46 allée d'Italie, 69364 Lyon Cedex 07, \\ FRANCE \\ Nicolas.Brisebarre@ens-lyon.fr, Jean-Michel.Muller@ens-lyon.fr
}

\begin{abstract}
We introduce an algorithm for multiplying a floatingpoint number $x$ by a constant $C$ that is not exactly representable in floating-point arithmetic. Our algorithm uses a multiplication and a fused multiply and add instruction. We give methods for checking whether, for a given value of $C$ and a given floating-point format, our algorithm returns a correctly rounded result for any $x$. When it does not, our methods give the values $x$ for which it does not.
\end{abstract}

\section{Introduction}

Many numerical algorithms require multiplications by constants that are not exactly representable in floating-point (FP) arithmetic. Typical constants that are used [1, 4] are $\pi, 1 / \pi, \ln (2), e, B_{k} / k$ ! (Euler-McLaurin summation), $\cos (k \pi / N)$ and $\sin (k \pi / N)$ (Fast Fourier Transforms). Some numerical integration formulas also naturally involve multiplications by constants.

For approximating $C x$, where $C$ is an infinite-precision constant and $x$ is an FP number, the desirable result would be the best possible one, namely $\circ(C x)$, where $\circ(u)$ is $u$ rounded to the nearest FP number. In practice one usually defines a constant $C_{h}$, equal to the FP number that is closest to $C$, and actually computes $C_{h} x$ (i.e., what is returned is $\left.\circ\left(C_{h} x\right)\right)$. The obtained result is frequently different from $\circ(C x)$ (see Section 1 for some statistics).

Our goal here is to be able - at least for some constants and some FP formats - to return $\circ(C x)$ for all input FP numbers $x$ (provided no overflow or underflow occur), and at a low cost (i.e., using a very few arithmetic operations only). To do that, we will use fused multiply and add instructions. The fused multiply and add instruction (FMA for short) is available on some current processors such as the IBM Power PC or the Intel/HP Itanium. It evaluates an expression $a x+b$ with one final rounding error only. This makes it possible to perform correctly rounded division using Newton-Raphson division [9, 3, 8]. Also, this makes evaluation of scalar products and polynomials faster and, generally, more accurate than with conventional (addition and multiplication) floating-point operations.

\section{Some statistics}

Let $n$ be the number of mantissa bits of the considered FP format (usual values of $n$ are 24, 53, 64, 113). For small values of $n$, one can compute $\circ\left(C_{h} x\right)$ and $\circ(C x)$ for all possible values of the mantissa of $x$. The obtained results are given in Table 1 , for $C=\pi$. They show that the "naive" method that consists in computing $\circ\left(C_{h} x\right)$ often returns an incorrectly rounded result (in around $41 \%$ of the cases for $n=7)$.

\section{The algorithm}

We want to compute $C x$ with correct rounding (assuming rounding to nearest even), where $C$ is a constant (i.e., $C$ is known at compile time). $C$ is not an FP number (otherwise the problem would be straightforward). We assume that a FMA instruction is available. We assume that the operands are stored in a binary FP format with $n$-bit mantissas. We also assume that the two following FP numbers are pre-computed:

$$
\left\{\begin{array}{l}
C_{h}=\circ(C) \\
C_{\ell}=\circ\left(C-C_{h}\right),
\end{array}\right.
$$

where $\circ(t)$ stands for $t$ rounded to the nearest FP number. 


\begin{tabular}{|r|l|}
\hline$n$ & $\begin{array}{c}|c| \\
\text { Proportion of } \\
\text { correctly rounded } \\
\text { results }\end{array}$ \\
\hline \hline 5 & 0.93750 \\
6 & 0.78125 \\
7 & 0.59375 \\
$\ldots$ & $\ldots$ \\
16 & 0.86765 \\
17 & 0.73558 \\
$\ldots$ & $\ldots$ \\
24 & 0.66805 \\
\hline
\end{tabular}

Table 1. Proportion of input values $x$ for which $\circ\left(C_{h} x\right)=\circ(C x)$ for $C=\pi$ and various values of the number $n$ of mantissa bits.

In the sequel of the paper, we analyze the behavior of the following algorithm. We aim at being able to know for which values of $C$ and $n$ it will return a correctly rounded result for any $x$. When it does not, we wish to know for which values of $x$ it does not.

\section{Algorithm 1 (Multiplication by $C$ with a multiplication and a FMA). From $x$, compute}

$$
\left\{\begin{array}{l}
u_{1}=\circ\left(C_{\ell} x\right) \\
u_{2}=\circ\left(C_{h} x+u_{1}\right) .
\end{array}\right.
$$

The result to be returned is $u_{2}$.

When $C$ is the exact reciprocal of an FP number, this algorithm coincides with an algorithm for division by a constant given in [2]. Obviously (provided no overflow/underflow occur) if Algorithm 1 gives a correct result with a given constant $C$ and a given input variable $x$, it will work as well with a constant $2^{p} C$ and an input variable $2^{q} x$, where $p$ and $q$ are integers. Also, if $x$ is a power of 2 or if $C$ is exactly representable (i.e., $C_{\ell}=0$ ), or if $C-C_{h}$ is a power of 2 (so that $u_{1}$ is exactly $\left(C-C_{h}\right) x$ ), it is straightforward to show that $u_{2}=\circ(C x)$. Hence, without loss of generality, we assume in the following that $1<x<2$ and $1<C<2$, that $C$ is not exactly representable, and that $C-C_{h}$ is not a power of 2 .

In Section 4, we give three methods. The first two ones either certify that Algorithm 1 always returns a correctly rounded result, or give a "bad case" (i.e., a number $x$ for which $u_{2} \neq \circ(C x)$ ), or are not able to infer anything. The third one is able to return all "bad cases", or certify that there are none. These methods use the following property, that bounds the maximum possible distance between $u_{2}$ and $C x$ in Algorithm 1. Of course, Algorithm 1 works for a given constant $C$ and precision $n$ if all floating-point values of $x$ are such that $\left|u_{2}-C x\right|<1 / 2$ ulp $\left(u_{2}\right)$. It is worth being noticed that without the use of a FMA instruction (that is, if Algorithm 1 was executed using ordinary FMUL and FADD), except for a few very simple values of the constant $C$ - e.g., powers of $2-$, Algorithm 1 would fail to return a correctly rounded result for all values of $x$.

\section{Property 1}

Define $x_{\text {cut }}=2 / C$ and

$$
\epsilon_{1}=\left|C-\left(C_{h}+C_{\ell}\right)\right|
$$

- If $x<x_{\text {cut }}$ then $\left|u_{2}-C x\right|<1 / 2$ ulp $\left(u_{2}\right)+\eta$,

- If $x \geq x_{\text {cut }}$ then $\left|u_{2}-C x\right|<1 / 2$ ulp $\left(u_{2}\right)+\eta^{\prime}$,

where

$$
\left\{\begin{array}{l}
\eta=\frac{1}{2} u l p\left(C_{\ell} x_{c u t}\right)+\epsilon_{1} x_{c u t} \\
\eta^{\prime}=u l p\left(C_{\ell}\right)+2 \epsilon_{1}
\end{array}\right.
$$

\section{Proof.}

From $1<C<2$ and $C_{h}=\circ(C)$, we deduce $\left|C-C_{h}\right|<$ $2^{-n}$, which gives (since $C-C_{h}$ is not a power of 2),

$$
\left|\epsilon_{1}\right| \leq \frac{1}{2} \operatorname{ulp}\left(C-C_{h}\right) \leq 2^{-2 n-1} .
$$

Now, we have,

$$
\begin{aligned}
\left|u_{2}-C x\right| \leq & \left|u_{2}-\left(C_{h} x+u_{1}\right)\right| \\
& +\left|\left(C_{h} x+u_{1}\right)-\left(C_{h} x+C_{\ell} x\right)\right| \\
& +\left|\left(C_{h}+C_{\ell}\right) x-C x\right| \\
\leq & \frac{1}{2} \operatorname{ulp}\left(u_{2}\right)+\left|u_{1}-C_{\ell} x\right|+\epsilon_{1}|x| \\
\leq & \frac{1}{2} \operatorname{ulp}\left(u_{2}\right)+\frac{1}{2} \operatorname{ulp}\left(C_{\ell} x\right)+\epsilon_{1}|x| .
\end{aligned}
$$

and $\frac{1}{2}$ ulp $\left(C_{\ell} x\right)+\epsilon_{1}|x|$ is less than $\frac{1}{2}$ ulp $\left(C_{\ell} x_{\text {cut }}\right)+\epsilon_{1}\left|x_{\text {cut }}\right|$ if $|x|<x_{\text {cut }}$ and less than ulp $\left(C_{\ell}\right)+2 \epsilon_{1}$ if $x_{\text {cut }} \leq x<2$.

If $\left|u_{2}-C x\right|$ is less than $1 / 2 \mathrm{ulp}\left(u_{2}\right)$, then $u_{2}$ is the FP number that is closest to $C x$. Hence our problem is to know if $C x$ can be at a distance larger than or equal to $\frac{1}{2}$ ulp $\left(u_{2}\right)$ from $u_{2}$. From (4), this would imply that $C x$ would be at a distance less than $\frac{1}{2}$ ulp $\left(C_{\ell} x\right)+\epsilon_{1}|x|<2^{-2 n+1}$ from the midpoint of two consecutive FP numbers (see Figure 1).

If $x<x_{\text {cut }}$ then $C x<2$, then the midpoint of two consecutive FP numbers around $C x$ is of the form $A / 2^{n}$, where $A$ is an odd integer between $2^{n}+1$ and $2^{n+1}-1$. If $x \geq x_{\text {cut }}$, then the midpoint of two consecutive FP numbers around $C x$ is of the form $A / 2^{n-1}$. For the sake of clarity of the proofs we assume that $x_{\text {cut }}$ is not an FP number (if $x_{\text {cut }}$ is an FP number, it suffices to separately check Algorithm 1 with $\left.x=x_{\text {cut }}\right)$. 


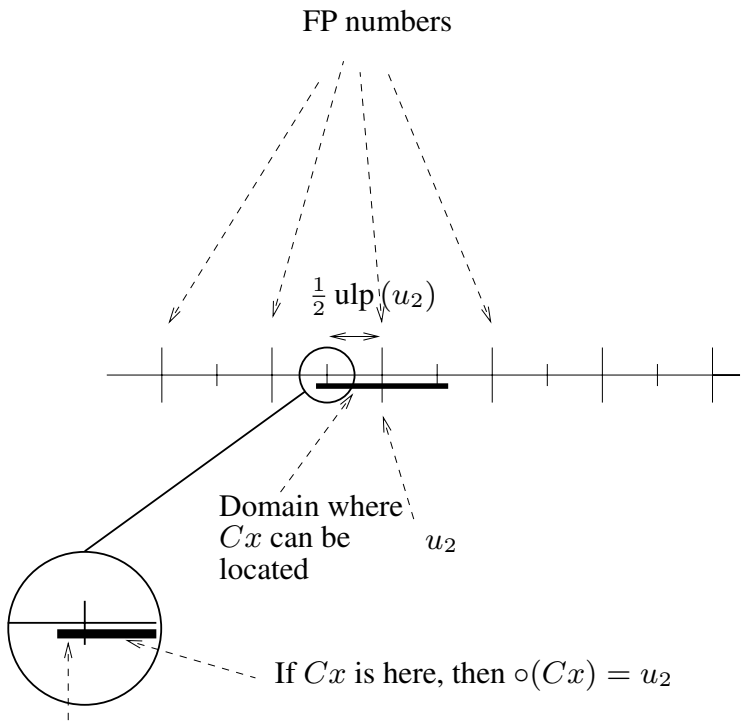

Can $C x$ be here?

Figure 1. From (4), we know that $C x$ is within $1 / 2$ ulp $\left(u_{2}\right)+\eta\left(\right.$ or $\left.\eta^{\prime}\right)$ from the FP number $u_{2}$, where $\eta$ is less than $2^{-2 n+1}$. If we can show that $C x$ cannot be at a distance less than or equal to $\eta$ (or $\eta^{\prime}$ ) from the midpoint of two consecutive floating-point numbers, then $u_{2}$ will be the FP number that is closest to $C x$.

\section{A reminder on continued fractions}

We just recall here the elementary results that we need in the following. For more information on continued fractions, see $[5,11,10,6]$.

Let $\alpha$ be a real number. From $\alpha$, consider the two sequences $\left(a_{i}\right)$ and $\left(r_{i}\right)$ defined by:

$$
\left\{\begin{array}{lll}
r_{0} & =\alpha \\
a_{i} & =\left\lfloor r_{i}\right\rfloor \\
r_{i+1} & = & \frac{1}{r_{i}-a_{i}}
\end{array}\right.
$$

If $\alpha$ is irrational, then these sequences are defined for any $i$ (i.e., $r_{i}$ is never equal to $a_{i}$ ), and the rational number

$$
\frac{p_{i}}{q_{i}}=a_{0}+\frac{1}{a_{1}+\frac{1}{a_{2}+\frac{1}{a_{3}+\frac{1}{\ddots \cdot+\frac{1}{a_{i}}}}}}
$$

is called the $i$ th convergent to $\alpha$. If $\alpha$ is rational, then these sequences finish for some $k$, and $p_{k} / q_{k}=\alpha$ exactly. The $p_{i} \mathrm{~s}$ and the $q_{i} \mathrm{~s}$ can be deduced from the $a_{i}$ using the following recurrences,

$$
\left\{\begin{array} { l } 
{ p _ { 0 } = a _ { 0 } , } \\
{ p _ { 1 } = a _ { 1 } a _ { 0 } + 1 , } \\
{ q _ { 0 } = 1 . }
\end{array} \quad \left\{\begin{array}{l}
q_{1}=a_{1}, \\
p_{n}=p_{n-1} a_{n}+p_{n-2}, \\
q_{n}=q_{n-1} a_{n}+q_{n-2} .
\end{array}\right.\right.
$$

The major interest of the continued fractions lies in the fact that $p_{i} / q_{i}$ is the best rational approximation to $\alpha$ among all rational numbers of denominator less than or equal to $q_{i}$.

We will use the following two results [5]

Theorem 1 Let $\left(p_{j} / q_{j}\right)_{j \geq 1}$ be the convergents of $\alpha$. For any $(p, q) \in \mathbb{Z} \times \mathbb{N}^{*}$, with $q<q_{n+1}$, we have

$$
|p-\alpha q| \geq\left|p_{n}-\alpha q_{n}\right| \text {. }
$$

Theorem 2 Let $p, q$ be nonzero integers, with $\operatorname{gcd}(p, q)=$ 1. If

$$
\left|\frac{p}{q}-\alpha\right|<\frac{1}{2 q^{2}}
$$

then $p / q$ is a convergent of $\alpha$.

\section{Three methods for analyzing Algorithm 1}

\subsection{Method 1: use of Theorem 1}

Define $X=2^{n-1} x$ and $X_{\text {cut }}=\left\lfloor 2^{n-1} x_{\text {cut }}\right\rfloor . X$ and $X_{\text {cut }}$ are integers between $2^{n-1}+1$ and $2^{n}-1$. We separate the cases $x<x_{\text {cut }}$ and $x>x_{\text {cut }}$.

\subsubsection{If $x<x_{\text {cut }}$}

We want to know if there is an integer $A$ between $2^{n}+1$ and $2^{n+1}-1$ such that

$$
\left|C x-\frac{A}{2^{n}}\right|<\eta
$$

where $\eta$ is defined in Property 1. (6) is equivalent to

$$
|2 C X-A|<2^{n} \eta
$$

Define $\left(p_{i} / q_{i}\right)_{i \geq 1}$ as the convergents of $2 C$. Let $k$ be the smallest integer such that $q_{k+1}>X_{\text {cut }}$, and define $\delta=$ $\left|p_{k}-2 C q_{k}\right|$. Theorem 1 implies that for any $A, X \in \mathbb{Z}$, with $0<X \leq X_{\text {cut }},|2 C X-A| \geq \delta$. Therefore

1. if $\delta \geq 2^{n} \eta$ then $\left|C x-A / 2^{n}\right|<\eta$ is impossible. In that case, Algorithm 1 returns a correctly rounded result for any $x<x_{\text {cut }}$;

2. if $\delta<2^{n} \eta$ then we try Algorithm 1 with $y=q_{k} 2^{-n+1}$. If the obtained result is not $\circ(y C)$, then we know that Algorithm 1 fails for at least one value ${ }^{1}$. Otherwise, we cannot infer anything.

\footnotetext{
${ }^{1}$ It is possible that $y$ be not between 1 and $x_{\text {cut }}$. It will anyway be a counterexample, i.e., an $n$-bit number for which Algorithm 1 fails.
} 


\subsubsection{If $x>x_{\text {cut }}$}

We want to know if there is an integer $A$ between $2^{n}+1$ and $2^{n+1}-1$ such that

$$
\left|C x-\frac{A}{2^{n-1}}\right|<\eta^{\prime}
$$

where $\eta^{\prime}$ is defined in Property 1. (8) is equivalent to

$$
|C X-A|<2^{n-1} \eta^{\prime}
$$

Define $\left(p_{i}^{\prime} / q_{i}^{\prime}\right)_{i \geq 1}$ as the convergents of $C$. Let $k^{\prime}$ be the smallest integer such that $q_{k^{\prime}+1}^{\prime} \geq 2^{n}$, and define $\delta^{\prime}=$ $\left|p_{k^{\prime}}^{\prime}-C q_{k^{\prime}}^{\prime}\right|$. Theorem 1 implies that for any $A, X \in \mathbb{Z}$, with $X_{\text {cut }} \leq X<2^{n},|C X-A| \geq \delta^{\prime}$. Therefore

1. if $\delta^{\prime} \geq 2^{n-1} \eta^{\prime}$ then $\left|C x-A / 2^{n-1}\right|<\eta^{\prime}$ is impossible. In that case, Algorithm 1 returns a correctly rounded result for any $x>x_{\text {cut }}$;

2. if $\delta^{\prime}<2^{n-1} \eta^{\prime}$ then we try Algorithm 1 with $y=$ $q_{k^{\prime}}^{\prime} 2^{-n+1}$. If the obtained result is not $\circ(y C)$, then we know that Algorithm 1 fails for at least one value. Otherwise, we cannot infer anything.

\subsection{Method 2: use of Theorem 2}

Again, we use $X=2^{n-1} x$ and $X_{\text {cut }}=\left\lfloor 2^{n-1} x_{\text {cut }}\right\rfloor$, and we separate the cases $x<x_{\text {cut }}$ and $x>x_{\text {cut }}$.

\subsubsection{If $x>x_{\text {cut }}$}

If

$$
\left|C x-\frac{A}{2^{n-1}}\right|<\epsilon_{1} x+\frac{1}{2} \operatorname{ulp}\left(C_{\ell} x\right)
$$

then,

$$
\left|C-\frac{A}{X}\right|<\epsilon_{1}+\frac{2^{n-2}}{X} \operatorname{ulp}\left(C_{\ell} x\right) .
$$

Now, if

$$
2^{2 n+1} \epsilon_{1}+2^{2 n-1} \operatorname{ulp}\left(2 C_{\ell}\right) \leq 1,
$$

then for any $X<2^{n}$ (i.e., $x<2$ ),

$$
\epsilon_{1}+\frac{2^{n-2}}{X} \operatorname{ulp}\left(C_{\ell} x\right)<\frac{1}{2 X^{2}} .
$$

Hence, if (11) is satisfied, then (10) implies (from Theorem 2) that $A / X$ is a convergent of $C$. This means that if (11) is satisfied, to find the possible bad cases for Algorithm 1 it suffices to examine the convergents of $C$ of denominator less than $2^{n}$. We can quickly eliminate most of them. A given convergent $p / q($ with $\operatorname{gcd}(p, q)=1)$ is a candidate for generating a value $X$ for which Algorithm 1 does not work if there exist $X=m q$ and $A=m p$ such that

$$
\left\{\begin{array}{l}
X_{\text {cut }}<X \leq 2^{n}-1, \\
2^{n}+1 \leq A \leq 2^{n+1}-1, \\
\left|\frac{C X}{2^{n-1}}-\frac{A}{2^{n-1}}\right|<\epsilon_{1} \frac{X}{2^{n-1}}+\frac{1}{2} \operatorname{ulp}\left(C_{\ell} x\right) .
\end{array}\right.
$$

This would mean

$$
\left|C \frac{m q}{2^{n-1}}-\frac{m p}{2^{n-1}}\right|<\epsilon_{1} \frac{m q}{2^{n-1}}+\frac{1}{2} \operatorname{ulp}\left(2 C_{\ell}\right),
$$

which would imply

$$
|C q-p|<\epsilon_{1} q+\frac{2^{n-1}}{m^{*}} \operatorname{ulp}\left(C_{\ell}\right),
$$

where $m^{*}=\left\lceil X_{\text {cut }} / q\right\rceil$ is the smallest possible value of $m$. Hence, if Condition (12) is not satisfied, convergent $p / q$ cannot generate a bad case for Algorithm 1.

Now, if Condition (12) is satisfied, we have to check Algorithm 1 with all values $X=m q$, with $m^{*} \leq m \leq$ $\left\lfloor\left(2^{n}-1\right) / q\right\rfloor$.

\subsubsection{If $x<x_{\text {cut }}$}

If

$$
\left|C x-\frac{A}{2^{n}}\right|<\epsilon_{1} x_{\text {cut }}+\frac{1}{2} \mathrm{ulp}\left(C_{\ell} x_{\text {cut }}\right)
$$

then

$$
\left|2 C-\frac{A}{X}\right|<2^{n} \times \frac{\epsilon_{1} x_{\mathrm{cut}}+\frac{1}{2} \mathrm{ulp}\left(C_{\ell} x_{\mathrm{cut}}\right)}{X} .
$$

Therefore, since $X \leq X_{\text {cut }}$, if

$$
\epsilon_{1} x_{\text {cut }}+\frac{1}{2} \operatorname{ulp}\left(C_{\ell} x_{\text {cut }}\right) \leq \frac{1}{2^{n+1} X_{\text {cut }}}
$$

then we can apply Theorem 2: if $\left|C x-A / 2^{n}\right|<\epsilon_{1} x_{\text {cut }}+$ $\frac{1}{2}$ ulp $\left(C_{\ell} x_{\text {cut }}\right)$ then $A / X$ is a convergent of $2 C$.

In that case, we have to check the convergents of $2 C$ of denominator less than or equal to $X_{\text {cut }}$. A given convergent $p / q($ with $\operatorname{gcd}(p, q)=1)$ is a candidate for generating a value $X$ for which Algorithm 1 does not work if there exist $X=m q$ and $A=m p$ such that

$$
\left\{\begin{array}{l}
2^{n-1} \leq X \leq X_{\mathrm{cut}} \\
2^{n}+1 \leq A \leq 2^{n+1}-1 \\
\left|\frac{C X}{2^{n-1}}-\frac{A}{2^{n}}\right|<\epsilon_{1} x_{\mathrm{cut}}+\frac{1}{2} \text { ulp }\left(C_{\ell} x_{\mathrm{cut}}\right) .
\end{array}\right.
$$

This would mean

$$
\left|C \frac{m q}{2^{n-1}}-\frac{m p}{2^{n}}\right|<\epsilon_{1} x_{\mathrm{cut}}+\frac{1}{2} \mathrm{ulp}\left(C_{\ell} x_{\mathrm{cut}}\right),
$$


which would imply

$$
<\frac{2^{n}}{m^{*}}\left(\epsilon_{1} x_{\text {cut }}+\frac{1}{2} \operatorname{ulp}\left(C_{\ell} x_{\text {cut }}\right)\right),
$$

where $m^{*}=\left\lceil 2^{n-1} / q\right\rceil$ is the smallest possible value of $m$. Hence, if (14) is not satisfied, convergent $p / q$ cannot generate a bad case for Algorithm 1.

Now, if (14) is satisfied, we have to check Algorithm 1 with all values $X=m q$, with $m^{*} \leq m \leq\left\lfloor X_{\text {cut }} / q\right\rfloor$.

This last result and (4) make it possible to deduce:

Theorem 3 (Conditions on $C$ and $n$ ) Assume $1<C<2$. Let $x_{\text {cut }}=2 / C$, and $X_{\text {cut }}=\left\lfloor 2^{n-1} x_{\text {cut }}\right\rfloor$.

- If $X=2^{n-1} x>X_{\text {cut }}$ and $2^{2 n+1} \epsilon_{1}+$ $2^{2 n-1}$ ulp $\left(2 C_{\ell}\right) \leq 1$ then Algorithm 1 will always return a correctly rounded result, except possibly if $X$ is a multiple of the denominator of a convergent $p / q$ of $C$ for which $|C q-p|<\epsilon_{1} q+\frac{2^{n-1}}{\left\lceil X_{\text {cut }} / q\right\rceil} u l p\left(C_{\ell}\right)$;

- if $X=2^{n-1} x \leq X_{\text {cut }}$ and $\epsilon_{1} x_{\text {cut }}+1 / 2$ ulp $\left(C_{\ell} x_{\text {cut }}\right) \leq$ $1 /\left(2^{n+1} X_{\text {cut }}\right)$ then Algorithm 1 will always return a correctly rounded result, except possibly if $X$ is a multiple of the denominator of $a$ convergent $p / q$ of $2 C$ for which $|2 C q-p|<$ $\frac{2^{n}}{\left\lceil 2^{n-1} / q\right\rceil}\left(\epsilon_{1} x_{c u t}+\frac{1}{2} u l p\left(C_{\ell} x_{c u t}\right)\right)$.

\subsection{Method 3: refinement of Method 2}

When Method 2 fails to return an answer, we can use the following method.

We have $\left|C-C_{h}\right|<2^{-n}$, hence ulp $\left(C_{\ell}\right) \leq 2^{-2 n}$.

\subsubsection{If $x<x_{\text {cut }}$}

if ulp $\left(C_{\ell}\right) \leq 2^{-2 n-2}$ then we have

$$
\left|u_{2}-C x\right|<\frac{1}{2} \operatorname{ulp}\left(u_{2}\right)+2^{-2 n-1} .
$$

For any integer $A$, the inequality

$$
\left|C x-\frac{2 A+1}{2^{n}}\right| \leq \frac{1}{2^{2 n+1}}
$$

implies

$$
|2 C X-2 A-1| \leq \frac{1}{2^{n+1}}<\frac{1}{2 X}:
$$

$(2 A+1) / X$ is a convergent of $2 C$ from Theorem 2 . It suffices then to check (as in Method 2) the convergents of $2 C$ of denominator less or equal to $X_{\text {cut }}$.
Now, assume ulp $\left(C_{\ell}\right) \geq 2^{-2 n-1}$. We have,

$$
-\operatorname{ulp}\left(C_{\ell}\right)+C_{\ell} \frac{X}{2^{n-1}} \leq u_{1} \leq \operatorname{ulp}\left(C_{\ell}\right)+C_{\ell} \frac{X}{2^{n-1}}
$$

i.e.,

$$
\begin{gathered}
-2^{2 n} \operatorname{ulp}\left(C_{\ell}\right)+2^{n+1} C_{\ell} X \\
\leq u_{1} 2^{2 n} \\
\leq 2^{2 n} \operatorname{ulp}\left(C_{\ell}\right)+2^{n+1} C_{\ell} X .
\end{gathered}
$$

We look for the integers $X, 2^{n-1} \leq X \leq X_{\text {cut }}$, such that there exists an integer $A, 2^{n-1} \leq A \leq 2^{n}-1$, with

$$
\left|C_{h} \frac{X}{2^{n-1}}+u_{1}-\frac{2 A+1}{2^{n}}\right|<2 \operatorname{ulp}\left(C_{\ell}\right)
$$

i.e.,

$$
\left|\frac{C_{h} X}{2^{n} \operatorname{ulp}\left(C_{\ell}\right)}+\frac{u_{1}}{2 \operatorname{ulp}\left(C_{\ell}\right)}-\frac{2 A+1}{2^{n+1} \operatorname{ulp}\left(C_{\ell}\right)}\right|<1 .
$$

Since $u_{1} /\left(2 \mathrm{ulp}\left(C_{\ell}\right)\right)$ is half an integer and $\frac{C_{h} X}{2^{n} \mathrm{ulp}\left(C_{\ell}\right)}$ and $\frac{2 A+1}{2^{n+1} \mathrm{ulp}\left(C_{\ell}\right)}$ are integers, we have

$$
\frac{C_{h} X}{2^{n} \operatorname{ulp}\left(C_{\ell}\right)}+\frac{u_{1}}{2 \operatorname{ulp}\left(C_{\ell}\right)}-\frac{2 A+1}{2^{n+1} \operatorname{ulp}\left(C_{\ell}\right)}=0, \pm 1 / 2 .
$$

Then, combining these three equations with inequalities (15), we get the following three pairs of inequalities

$$
\begin{gathered}
0 \leq 2 X\left(C_{h}+C_{\ell}\right)-(2 A+1)+2^{n} \text { ulp }\left(C_{\ell}\right) \\
\leq 2^{n+1} \operatorname{ulp}\left(C_{\ell}\right),
\end{gathered}
$$

$$
\begin{gathered}
0 \leq 2 X\left(C_{h}+C_{\ell}\right)-(2 A+1) \\
\leq 2^{n+1} \operatorname{ulp}\left(C_{\ell}\right)
\end{gathered}
$$

$$
\begin{gathered}
0 \leq 2 X\left(C_{h}+C_{\ell}\right)-(2 A+1)+2^{n+1} \mathrm{ulp}\left(C_{\ell}\right) \\
\leq 2^{n+1} \mathrm{ulp}\left(C_{\ell}\right) .
\end{gathered}
$$

For $y \in \mathbb{R}$, let $\{y\}$ be the fractional part of $y:\{y\}=$ $y-\lfloor y\rfloor$. These three inequalities can be rewritten as

$$
\left\{2 X\left(C_{h}+C_{\ell}\right)+2^{n} \operatorname{ulp}\left(C_{\ell}\right)\right\} \leq 2^{n+1} \operatorname{ulp}\left(C_{\ell}\right),
$$

$$
\begin{gathered}
\left\{2 X\left(C_{h}+C_{\ell}\right)\right\} \leq 2^{n+1} \operatorname{ulp}\left(C_{\ell}\right), \\
\left\{2 X\left(C_{h}+C_{\ell}\right)+2^{n+1} \operatorname{ulp}\left(C_{\ell}\right)\right\} \leq 2^{n+1} \operatorname{ulp}\left(C_{\ell}\right) .
\end{gathered}
$$

We use an efficient algorithm due to V. Lefèvre [7] to determine the integers $X$ solution of each inequality. 


\subsubsection{If $x>x_{\text {cut }}$}

if ulp $\left(C_{\ell}\right) \leq 2^{-2 n-1}$ then we have

$$
\left|u_{2}-C x\right|<\frac{1}{2} \operatorname{ulp}\left(u_{2}\right)+2^{-2 n} .
$$

Therefore, for any integer $A$, the inequality

$$
\left|C x-\frac{2 A+1}{2^{n-1}}\right| \leq \frac{1}{2^{2 n}}
$$

is equivalent to

$$
|C X-2 A-1| \leq \frac{1}{2^{n+1}}<\frac{1}{2 X},
$$

$(2 A+1) / X$ is necessarily a convergent of $C$ from Theorem 2. It suffices then to check, as indicated in Method 2, the convergents of $C$ of denominator less or equal to $2^{n}-1$.

Now, assume ulp $\left(C_{\ell}\right)=2^{-2 n}$. We look for the integers $X, X_{\text {cut }}+1 \leq X \leq 2^{n}-1$, such that there exists an integer $A, 2^{n-1} \leq A \leq 2^{n}-1$, with

$$
\left|C_{h} \frac{X}{2^{n-1}}+u_{1}-\frac{2 A+1}{2^{n-1}}\right|<\frac{1}{2^{2 n}}
$$

i.e.,

$$
\left|2^{n+1} C_{h} X+u_{1} 2^{2 n}-2^{n+1}(2 A+1)\right|<1 .
$$

Since $u_{1} 2^{2 n}, 2^{n+1} C_{h} X$ and $2^{n+1}(2 A+1) \in \mathbb{Z}$, we have

$$
2^{n+1} C_{h} X+u_{1} 2^{2 n}-2^{n}(2 A+1)=0 .
$$

Then, combining this equation with inequalities (15), we get the inequalities

$$
0 \leq X\left(C_{h}+C_{\ell}\right)-(2 A+1)+\frac{1}{2^{n+1}} \leq \frac{1}{2^{n}},
$$

that is to say

$$
\left\{X\left(C_{h}+C_{\ell}\right)+\frac{1}{2^{n+1}}\right\} \leq \frac{1}{2^{n}} .
$$

Here again, we use Lefèvre's algorithm [7] to determine the integers $X$ solution of this inequality.

\section{Examples}

\subsection{Example 1: multiplication by $\pi$ in double pre- cision}

Consider the case $C=\pi / 2$ (which corresponds to multiplication by any number of the form $2^{ \pm j} \pi$ ), and $n=53$ (double precision), and assume we use Method 1. We find:

$$
\begin{cases}C_{h} & =884279719003555 / 562949953421312 \\ C_{\ell} & =6.123233996 \cdots \times 10^{-17} \\ \epsilon_{1} & =1.497384905 \cdots \times 10^{-33} \\ x_{\text {cut }} & =1.2732395447351626862 \cdots \\ \operatorname{ulp}\left(C_{\ell} x_{\mathrm{cut}}\right) & =2^{-106} \\ \operatorname{ulp}\left(C_{\ell}\right) & =2^{-106}\end{cases}
$$

Hence,

$$
\left\{\begin{array}{ll}
2^{n} \eta & =7.268364390 \times 10^{-17} \\
2^{n-1} \eta^{\prime} & =6.899839541 \times 10^{-17}
\end{array} .\right.
$$

Computing the convergents of $2 C$ and $C$ we find

$$
\frac{p_{k}}{q_{k}}=\frac{6134899525417045}{1952799169684491}
$$

and $\delta=9.495905771 \times 10^{-17}>2^{n} \eta$ (which means that Algorithm 1 works for $x<x_{\text {cut }}$ ), and

$$
\frac{p_{k^{\prime}}^{\prime}}{q_{k^{\prime}}^{\prime}}=\frac{12055686754159438}{7674888557167847}
$$

and $\delta^{\prime}=6.943873667 \times 10^{-17}>2^{n-1} \eta^{\prime}$ (which means that Algorithm 1 works for $x>x_{\text {cut }}$ ). We therefore deduce:

\section{Theorem 4 (Correctly rounded multiplication by $\pi$ )}

Algorithm 1 always returns a correctly rounded result in double precision with $C=2^{j} \pi$, where $j$ is any integer, provided no under/overflow occur.

Hence, in that case, multiplying by $\pi$ with correct rounding only requires 2 consecutive FMAs.

\subsection{Example 2: multiplication by $\ln (2)$ in double precision}

Consider the case $C=2 \ln (2)$ (which corresponds to multiplication by any number of the form $2^{ \pm j} \ln (2)$ ), and $n=53$, and assume we use Method 2. We find:

$$
\begin{cases}C_{h} & =\frac{6243314768165359}{4503599627370496}, \\ C_{\ell} & =4.638093628 \cdots \times 10^{-17}, \\ x_{\text {cut }} & =1.442695 \cdots \\ \epsilon_{1} & =1.141541688 \cdots \times 10^{-33}, \\ \epsilon_{1} x_{\text {cut }} & \\ +\frac{1}{2} \text { ulp }\left(C_{\ell} x_{\text {cut }}\right) & =7.8099 \cdots \times 10^{-33} \\ 1 /\left(2^{n+1} X_{\text {cut }}\right) & =8.5437 \cdots \times 10^{-33} .\end{cases}
$$

Since $\epsilon_{1} x_{\text {cut }}+1 / 2$ ulp $\left(C_{\ell} x_{\text {cut }}\right) \leq 1 /\left(2^{n+1} X_{\text {cut }}\right)$, to find the possible bad cases for Algorithm 1 that are less than $x_{\text {cut }}$, it suffices to check the convergents of $2 C$ of denominator less than or equal to $X_{\text {cut }}$. These convergents are:

$2,3,11 / 4,25 / 9,36 / 13,61 / 22,890 / 321,2731 / 985$, $25469 / 9186,1097898 / 395983,1123367 / 405169$,

$2221265 / 801152,16672222 / 6013233,18893487 / 6814385$, $35565709 / 12827618,125590614 / 45297239$

$161156323 / 58124857,609059583 / 219671810$,

$1379275489 / 497468477$, 1988335072/717140287,

$5355945633 / 1931749051,7344280705 / 2648889338$,

$27388787748 / 9878417065,34733068453 / 12527306403$,

$62121856201 / 22405723468,96854924654 / 34933029871$,

$449541554817 / 162137842952$,

$2794104253556 / 1007760087583$

$3243645808373 / 1169897930535$

$6037750061929 / 2177658018118$

$39470146179947 / 14235846039243$,

$124448188601770 / 44885196135847$

$163918334781717 / 59121042175090$,

$288366523383487 / 104006238310937$,

$6219615325834944 / 2243252046704767$. 
None of them satisfies condition (14). Therefore there are no bad cases less than $x_{\text {cut }}$. Processing the case $x>x_{\text {cut }}$ is similar and gives the same result, hence:

Theorem 5 (Correctly rounded multiplication by $\ln (2)$ ) Algorithm 1 always returns a correctly rounded result in double precision with $C=2^{j} \ln (2)$, where $j$ is any integer, provided no under/overflow occur.

\subsection{Example 3: multiplication by $1 / \pi$ in double precision}

Consider the case $C=4 / \pi$ and $n=53$, and assume we use Method 1. We find:

$$
\begin{cases}C_{h} & =\frac{5734161139222659}{450359627370496}, \\ C_{\ell} & =-7.871470670 \cdots \times 10^{-17}, \\ \epsilon_{1} & =4.288574513 \cdots \times 10^{-33}, \\ x_{\text {cut }} & =1.570796 \cdots, \\ C_{\ell} x_{\text {cut }} & =-1.236447722 \cdots \times 10^{-16}, \\ \operatorname{ulp}\left(C_{\ell} x_{\text {cut }}\right) & =2^{-105}, \\ 2^{n} \eta & =1.716990939 \cdots \times 10^{-16}, \\ p_{k} / q_{k} & =\frac{15486085235905811}{6081371451248382}, \\ \delta & =7.669955467 \cdots \times 10^{-17} .\end{cases}
$$

Consider the case $x<x_{\text {cut }}$. Since $\delta<2^{n} \eta$, there can be bad cases for Algorithm 1. We try Algorithm 1 with $X$ equal to the denominator of $p_{k} / q_{k}$, that is, 6081371451248382 , and we find that it does not return $\circ(c X)$ for that value. Hence, there is at least one value of $x$ for which Algorithm 1 does not work.

Method 3 certifies that $X=6081371451248382$, i.e., $6081371451248382 \times 2^{ \pm k}$ are the only FP values for which Algorithm 1 fails.

\subsection{Example 4: multiplication by $\sqrt{2}$ in single pre- cision}

Consider the case $C=\sqrt{2}$, and $n=24$ (which corresponds to single precision), and assume we use Method 1 . We find:

$$
\begin{cases}C_{h} & =11863283 / 8388608 \\ C_{\ell} & =2.420323497 \cdots \times 10^{-8} \\ \epsilon_{1} & =7.628067479 \cdots \times 10^{-16} \\ X_{\text {cut }} & =11863283 \\ \text { ulp }\left(C_{\ell} x_{\text {cut }}\right) & =2^{-48} \\ 2^{n} \eta & =4.790110735 \cdots \times 10^{-8} \\ p_{k} / q_{k} & =22619537 / 7997214 \\ \delta & =2.210478490 \cdots \times 10^{-8} \\ 2^{n-1} \eta^{\prime} & =2.769893477 \cdots \times 10^{-8} \\ p_{k^{\prime}} / q_{k^{\prime}} & =22619537 / 15994428 \\ \delta^{\prime} & =2.210478490 \cdots \times 10^{-8}\end{cases}
$$

Since $2^{n} \eta>\delta$ and $X=q_{k}=7997214$ is not a bad case, we cannot infer anything in the case $x<x_{\text {cut }}$. Also, since $2^{n-1} \eta^{\prime}>\delta^{\prime}$ and $X=q_{k^{\prime}}=15994428$ is not a bad case, we cannot infer anything in the case $x \geq x_{\text {cut }}$. Hence, in the case $C=\sqrt{2}$ and $n=24$, Method 1 does not allow us to know if the multiplication algorithm works for any input FP number $x$. In that case, Method 2 also fails. And yet, Method 3 or exhaustive testing (which is possible since $n=24$ is reasonably small) show that Algorithm 1 always works.

\section{Implementation and results}

As the reader will have guessed from the previous examples, using our Methods by paper and pencil calculation is fastidious and error-prone. We have written Maple programs that implement Methods 1 and 2, and a GP/PARI ${ }^{2}$ program that implements Method 3. They allow any user to quickly check, for a given constant $C$ and a given number $n$ of mantissa bits, if Algorithm 1 works for any $x$, and Method 3 gives all values of $x$ for which it does not work (if there are such values). These programs can be downloaded from the url

http: //perso.ens-lyon. fr/jean-michel. muller/MultConstant.html

These programs, along with some examples, are given in the appendix. Table 2 presents some obtained results. They show that implementing Method 1, Method 2 and Method 3 is necessary: Methods 1 and 2 do not return a result (either a bad case, or the fact that Algorithm 1 always works) for the same values of $C$ and $n$. For instance, in the case $C=\pi / 2$ and $n=53$, we know thanks to Method 1 that the multiplication algorithm always works, whereas Method 2 fails to give an answer. On the contrary, in the case $C=1 / \ln (2)$ and $n=24$, Method 1 does not give an answer, whereas Method 2 makes it possible to show that the algorithm always works. Method 3 always returns an answer, but is more complicated to implement: this is not a problem for getting in advance a result such as Theorem 4, for a general constant $C$. And yet, this might make method 3 difficult to implement in a compiler, to decide at compile-time if we can use our algorithm.

\section{Conclusion}

The three methods we have proposed allow one to check whether correctly rounded multiplication by an "infinite precision" constant $C$ is feasible at a low cost (one multiplication and one FMA). For instance, in double precision arithmetic, we can multiply by $\pi$ or $\ln (2)$ with correct rounding. Interestingly enough, although it is always possible to build ad hoc values of $C$ for which Algorithm 1 fails, for "general" values of $C$, our experiments show that Algorithm 1 works for most values of $n$.

\footnotetext{
${ }^{2}$ http://pari.math.u-bordeaux.fr/
} 


\begin{tabular}{|c|c|c|c|c|}
\hline$C$ & $n$ & method 1 & method 2 & method 3 \\
\hline$\pi$ & 8 & $\begin{array}{c}\text { Does not } \\
\text { work for } \\
226\end{array}$ & $\begin{array}{c}\text { Does not } \\
\text { work for } \\
226\end{array}$ & $\begin{array}{c}\mathrm{AW}(\mathrm{c}) \\
\text { unless } X= \\
226\end{array}$ \\
\hline$\pi$ & 24 & unable & unable & AW \\
\hline$\pi$ & 53 & AW & unable & AW \\
\hline$\pi$ & 64 & unable & AW & $\mathrm{AW}(\mathrm{c})$ \\
\hline$\pi$ & 113 & AW & $\mathrm{AW}$ & $\mathrm{AW}(\mathrm{c})$ \\
\hline $1 / \pi$ & 24 & unable & unable & AW \\
\hline $1 / \pi$ & 53 & $\begin{array}{c}\text { Does not } \\
\text { work for } \\
6081371451248382 \\
\end{array}$ & unable & $\begin{array}{c}\text { AW } \\
\text { unless } X= \\
6081371451248382\end{array}$ \\
\hline $1 / \pi$ & 64 & AW & AW & $\mathrm{AW}(\mathrm{c})$ \\
\hline $1 / \pi$ & 113 & unable & unable & AW \\
\hline $\ln 2$ & 24 & AW & AW & $\mathrm{AW}(\mathrm{c})$ \\
\hline $\ln 2$ & 53 & AW & unable & $\mathrm{AW}(\mathrm{c})$ \\
\hline $\ln 2$ & 64 & AW & unable & $\mathrm{AW}(\mathrm{c})$ \\
\hline $\ln 2$ & 113 & AW & AW & $\mathrm{AW}(\mathrm{c})$ \\
\hline$\frac{1}{\ln 2}$ & 24 & unable & AW & $\mathrm{AW}(\mathrm{c})$ \\
\hline$\frac{1}{\ln 2}$ & 53 & AW & AW & AW (c) \\
\hline$\frac{1}{\ln 2}$ & 64 & unable & unable & AW \\
\hline$\frac{1}{\ln 2}$ & 113 & unable & unable & AW \\
\hline $\ln 10$ & 24 & unable & AW & $\mathrm{AW}(\mathrm{c})$ \\
\hline $\ln 10$ & 53 & unable & unable & AW \\
\hline $\ln 10$ & 64 & unable & $\mathrm{AW}$ & $\mathrm{AW}(\mathrm{c})$ \\
\hline $\ln 10$ & 113 & AW & AW & $\mathrm{AW}(\mathrm{c})$ \\
\hline$\frac{1}{\ln 10}$ & 24 & unable & unable & AW \\
\hline$\frac{1}{\ln 10}$ & 53 & unable & AW & $\mathrm{AW}(\mathrm{c})$ \\
\hline$\frac{1}{\ln 10}$ & 64 & unable & AW & AW (c) \\
\hline$\frac{1}{\ln 10}$ & 113 & unable & unable & AW \\
\hline $\cos \frac{\pi}{8}$ & 24 & unable & unable & AW \\
\hline $\cos \frac{\pi}{8}$ & 53 & AW & AW & $\mathrm{AW}(\mathrm{c})$ \\
\hline $\cos \frac{\pi}{8}$ & 64 & AW & unable & AW \\
\hline $\cos \frac{\pi}{8}$ & 113 & unable & AW & $\mathrm{AW}(\mathrm{c})$ \\
\hline
\end{tabular}

\section{References}

[1] M. Abramowitz and I. A. Stegun. Handbook of mathematical functions with formulas, graphs and mathematical tables. Applied Math. Series 55. National Bureau of Standards, Washington, D.C., 1964.

[2] N. Brisebarre, J.-M. Muller, and S. Raina. Accelerating correctly rounded floating-point division when the divisor is known in advance. IEEE Transactions on Computers, 53(8):1069-1072, Aug. 2004.

[3] M. A. Cornea-Hasegan, R. A. Golliver, and P. Markstein. Correctness proofs outline for newton-raphson based floating-point divide and square root algorithms. In Koren and Kornerup, editors, Proceedings of the 14th IEEE Symposium on Computer Arithmetic (Adelaide, Australia), pages 96-105, Los Alamitos, CA, Apr. 1999. IEEE Computer Society Press.

[4] B. P. Flannery, W. H. Press, S. A. Teukolsky, and W. T. Vetterling. Numerical recipes in C. Cambridge University Press, 2 edition, 1992.

[5] G. H. Hardy and E. M. Wright. An introduction to the theory of numbers. Oxford University Press, 1979.

[6] A. Y. Khinchin. Continued Fractions. Dover, New York, 1997.

[7] V. Lefèvre. Developments in Reliable Computing, chapter An Algorithm That Computes a Lower Bound on the Distance Between a Segment and $\mathbb{Z}^{2}$, pages 203-212. Kluwer, Dordrecht, Netherlands, 1999.

[8] P. Markstein. IA-64 and Elementary Functions : Speed and Precision. Hewlett-Packard Professional Books. Prentice Hall, 2000. ISBN: 0130183482.

[9] P. W. Markstein. Computation of elementary functions on the IBM risc system/6000 processor. IBM Journal of Research and Development, 34(1):111-119, Jan. 1990.

[10] O. Perron. Die Lehre von den Kettenbruchen, 3. verb. und erweiterte Aufl. Teubner, Stuttgart, 1954-57.

[11] H. M. Stark. An Introduction to Number Theory. MIT Press, Cambridge, MA, 1981.

Table 2. Some results obtained using methods 1,2 and 3 . The results given for constant $C$ hold for all values $2^{ \pm j} C$. "AW" means "always works" and "unable" means "the method is unable to conclude". For method 3, "(c)" means that we have needed to check the convergents. 